\title{
A Simple Factor in Canonical Quantization Yields Affine Quantization Even for Quantum Gravity
}

\author{
John R. Klauder \\ Department of Physics and Department of Mathematics, University of Florida, Gainesville, US \\ Email:klauder@ufl.edu
}

How to cite this paper: Klauder, J.R. (2021) A Simple Factor in Canonical Quantization Yields Affine Quantization Even for Quantum Gravity. Journal of High Energy Physics, Gravitation and Cosmology, 7, 13281332 .

https://doi.org/10.4236/jhepgc.2021.74082

Received: August 13, 2021

Accepted: October 6, 2021

Published: October 9, 2021

Copyright $\odot 2021$ by author(s) and Scientific Research Publishing Inc. This work is licensed under the Creative Commons Attribution International License (CC BY 4.0). http://creativecommons.org/licenses/by/4.0/

\begin{abstract}
Canonical quantization (CQ) is built around $[Q, P]=i \hbar 1$, while affine quantization (AQ) is built around $[Q, D]=i \hbar Q$, where $D \equiv(P Q+Q P) / 2$. The basic CQ operators must fit $-\infty<P, Q<\infty$, while the basic AQ operators can fit $-\infty<P<\infty$ and $0<Q<\infty,-\infty<Q<0$, or even $-\infty<Q \neq 0<\infty$. AQ can also be the key to quantum gravity, as our simple outline demonstrates.
\end{abstract}

\section{Keywords}

Canonical Quantization (CQ), Affine Quantization (AQ), Quantum Gravity

\section{Canonical Quantization to Affine Quantization: CQ to AQ}

The CQ equation $[Q, P]=Q P-P Q=i \hbar 1$ is multiplied by $Q$ to give

$$
\begin{aligned}
& \{Q(Q P-P Q)+(Q P-P Q) Q\} / 2=\left\{Q^{2} P+Q P Q-Q P Q-P Q^{2}\right\} / 2 . \\
& =[Q,(Q P+P Q) / 2] \equiv[Q, D]=i \hbar Q
\end{aligned} .
$$

This principal equation requires that $Q \neq 0$, leading to the last expression which applies for $Q>0, Q<0$, or both. From a classical promotion viewpoint for $\mathrm{CQ}$ it is $-\infty<p \rightarrow P, q \rightarrow Q<\infty$, while for AQ it is $-\infty<d \equiv p q \rightarrow D \equiv(P Q+Q P) / 2<\infty$, while $-\infty<q \neq 0 \rightarrow Q \neq 0<\infty$. Note: $P^{\dagger} \neq P$, but $P^{\dagger} Q=P Q$.

Not every set of classical variables lead to valid quantum operators, neither for $\mathrm{CQ}$ nor for AQ. The correct choice of classical variables to promote quantum operators for CQ is Cartesian coordinates, which create a constant vanishing curvature. The classical coordinates that create a constant negative curvature ${ }^{1}$ are ${ }^{1} \mathrm{~A}$ constant negative curvature is discussed in [1]. 
the correct coordinates to promote quantum operators for AQ. ${ }^{2}$ Regarding CQ and $A Q$ operators, the principal difference is that CQ operators must span the whole real line, while the AQ operator $\mathrm{Q}$ strictly spans all positive coordinates, all negative coordinates, or both, provided $Q \neq 0 .{ }^{3}$

Different sets of classical problems require valid CQ or AQ quantizations. For example, a traditional harmonic oscillator, with $-\infty<p, q<\infty$, employs CQ, while a half-harmonic oscillator, with $-\infty<p<\infty$, but $0<q<\infty$, employs AQ [2]. Partial-harmonic oscillators, with $-\infty<p<\infty$, while $-b<q<\infty$ and $0<b<\infty$, also employ AQ [3].

Examples from scalar field theories show that $\varphi_{3}^{12}$ leads to "free" results for CQ while "non-free" results for AQ [4], and also "free" results for $\varphi_{4}^{4}$ [5] [6] [7] while AQ leads to a "non-free" result [8]. It becomes clear that CQ is not the only quantization procedure for various classical systems! The author has even suggested that quantum field theory might benefit by adding AQ to the usual procedures [9].

Regarding gravity, an affine result can offer a strictly positive metric, i.e., $\mathrm{d} s(x)^{2}=g_{a b}(x) \mathrm{d} x^{a} \mathrm{~d} x^{b}>0$, provided $\mathrm{d} x^{c} \not \equiv 0$. This ability offers a strong contribution to the quantization of gravity, as sketched below.

\section{The Essence of Quantum Gravity}

The author has shown that an affine quantization is the natural procedure to quantize Einstein's gravity, e.g., [10] [11]. Here we offer an easy and straightforward derivation that features the highlights of a natural quantum gravity. Our procedure is based on the analysis of other quantum procedures that lead toward a conventual Hamiltonian as part of a standard Schrödinger's representation followed by a suitable version of Schrödinger's equation.

\subsection{Classical Gravity}

The classical Hamiltonian, according to ADM [12], uses the metric $g_{a b}(x)=g_{b a}(x)$ and its determinant $g(x) \equiv \operatorname{det}\left[g_{a b}(x)\right],{ }^{4}$ the momentum $\pi^{c d}(x)=\pi^{d c}(x)$, or more useful, the momentric ${ }^{5} \pi_{d}^{c}(x) \equiv \pi^{c e}(x) g_{d e}(x)$, all of which leads to

$$
\begin{aligned}
& H\left(\pi_{b}^{a}, g_{c d}\right) \\
& =\int\left\{g(x)^{-1 / 2}\left[\pi_{b}^{a}(x) \pi_{a}^{b}(x)-\frac{1}{2} \pi_{a}^{a}(x) \pi_{b}^{b}(x)\right]+g(x)^{1 / 2} R(x)\right\} \mathrm{d}^{3} x,
\end{aligned}
$$

where $R(x)$ is the Ricci scalar [13]. The Poisson brackets for the momentric and metric fields are given by

\footnotetext{
${ }^{2}$ Classical variables for spin variables that form a constant positive curvature are the final set of correct classical variables that promote to valid quantum operators. The constant $(+, 0,-1)$ curvatures are all addressed in [2], Sec. 1.

${ }^{3}$ Instead of $-\infty<Q \neq 0<\infty$, we can also have $-\infty<Q \neq b<\infty$, provided $b$ is finite.

${ }^{4}$ The additional symbol $\{g\}$ stands for $\left\{g_{a b}(\cdot)>0\right\}$.

${ }^{5}$ The name momentric is taken from momentum and metric.
} 


$$
\begin{aligned}
& \left\{\pi_{b}^{a}(x), \pi_{d}^{c}\left(x^{\prime}\right)\right\}=\frac{1}{2} \delta^{3}\left(x, x^{\prime}\right)\left[\delta_{d}^{a} \pi_{b}^{c}(x)-\delta_{b}^{c} \pi_{d}^{a}(x)\right], \\
& \left\{g_{a b}(x), \pi_{d}^{c}\left(x^{\prime}\right)\right\}=\frac{1}{2} \delta^{3}\left(x, x^{\prime}\right)\left[\delta_{a}^{c} g_{b d}(x)+\delta_{b}^{c} g_{a d}(x)\right], \\
& \left\{g_{a b}(x), g_{c d}\left(x^{\prime}\right)\right\}=0 .
\end{aligned}
$$

Observe that these Poisson brackets are valid even if we change $g_{a b}(x)$ to $-g_{a b}(x)$, a property that allows us to retain only $g_{a b}(x)>0$. This is not possible with the Poisson brackets for canonical variables.

\subsection{Quantum Variables}

Passing to operator commutations by a promotion of the set of Poisson brackets to operator commutations leads, as established in [11], to

$$
\begin{aligned}
& {\left[\hat{\pi}_{b}^{a}(x), \hat{\pi}_{d}^{c}\left(x^{\prime}\right)\right]=i \frac{1}{2} \hbar \delta^{3}\left(x, x^{\prime}\right)\left[\delta_{d}^{a} \hat{\pi}_{b}^{c}(x)-\delta_{b}^{c} \hat{\pi}_{d}^{a}(x)\right],} \\
& {\left[\hat{g}_{a b}(x), \hat{\pi}_{d}^{c}\left(x^{\prime}\right)\right]=i \frac{1}{2} \hbar \delta^{3}\left(x, x^{\prime}\right)\left[\delta_{a}^{c} \hat{g}_{b d}(x)+\delta_{b}^{c} \hat{g}_{a d}(x)\right],} \\
& {\left[\hat{g}_{a b}(x), \hat{g}_{c d}\left(x^{\prime}\right)\right]=0 .}
\end{aligned}
$$

As with the Poisson brackets, these commutators are valid if we change $\hat{g}_{a b}(x)$ to $-\hat{g}_{a b}(x)$. For the momentric and metric operators, we again find that we can retain only $\hat{g}_{a b}(x)>0$. Moreover, with suitable regularization, it follows that $\hat{\pi}_{b}^{a}(x) \hat{g}(x)^{-1 / 2}=0$, which is proved in [11].

\subsection{Schrödinger's Representation and Equation}

Schrödinger's representation chooses $\hat{g}_{a b}(x)=g_{a b}(x)$ and

$$
\hat{\pi}_{d}^{c}(x)=-i \frac{1}{2} \hbar\left[g_{d e}(x)\left(\partial / \partial g_{c e}(x)\right)+\left(\partial / \partial g_{c e}(x)\right) g_{d e}(x)\right],
$$

which is well evaluated using a suitable regularization [11]. Finally, we are led to Schrödinger's equation

$$
\begin{aligned}
i \hbar \partial \Psi(\{g\}, t) / \partial t= & \left\{\int \left\{\left[\hat{\pi}_{b}^{a}(x) g(x)^{-1 / 2} \hat{\pi}_{a}^{b}(x)-\frac{1}{2} \hat{\pi}_{a}^{a}(x) g(x)^{-1 / 2} \hat{\pi}_{b}^{b}(x)\right]\right.\right. \\
& \left.\left.+g(x)^{1 / 2} R(x)\right\} \mathrm{d}^{3} x\right\} \Psi(\{g\}, t) .
\end{aligned}
$$

\subsection{Potential Wave Functions}

The expression $\hat{\pi}_{b}^{a}(x) g(x)^{-1 / 2}=0$ points to a form of wave function given by $\Psi(\{g\})=W(\{g\}) \Pi_{y} g(y)^{-1 / 2}$. The wave functions for the Hamiltonian are continuous in the metrics that make up $\{g\}$, which is natural for a Hamiltonian that contains spatial derivatives of the metric fields and enforces continuity within the Ricci scalar, $R(x)$.

The normalization of such wave functions may be given by

$$
\int|\Psi(\{g\})|^{2} \mathcal{D}\{g\}=\int|W(\{g\})|^{2} \Pi_{y} g(y)^{-1} \mathcal{D}\{g\}=1,
$$


while a matrix expression for an operator $A$ may be given by

$$
\begin{aligned}
& \int \Psi^{\prime}\left(\left\{g^{\prime}\right\}\right)^{*} A\left(\left\{g^{\prime}\right\},\{g\}\right) \Psi(\{g\}) \mathcal{D}\left\{g^{\prime}\right\} \mathcal{D}\{g\} \\
& =\int \Pi_{y^{\prime}} g^{\prime}\left(y^{\prime}\right)^{-1 / 2} A\left(\left\{g^{\prime}\right\},\{g\}\right) \Pi_{y} g(y)^{-1 / 2} \mathcal{D}\left\{g^{\prime}\right\} \mathcal{D}\{g\} .
\end{aligned}
$$

It may be noticed that the factors $\Pi_{y} g(y)^{-1 / 2}$ act somewhat like coherent states', e.g., [14], in these equations.

\section{Conclusions}

The forgoing study is focused on the gravity Hamiltonian, which is the most difficult aspect of quantum gravity. Additional topics, such as fulfilling constraints, are examined in [11], along with a somewhat different viewpoint in [2]. An earlier paper [15] is especially focused on establishing a path through the multiple constraints that quantum gravity faces.

Contributions by other researchers using affine procedures to quantize gravity are welcome to add to the present story.

\section{Conflicts of Interest}

The author declares no conflicts of interest regarding the publication of this paper.

\section{References}

[1] Wikipedia. Constant Negative Curvature. Hyperbolic Dynamics, Scholarpedia.

[2] Gouba, L. (2021) Affine Quantization on the Half Line. Journal of High Energy Physics, Gravitation and Cosmology, 7, 352-365. https://doi.org/10.4236/jhepgc.2021.71019

[3] Handy, C. and Klauder, J. (2021) Proof That Half-Harmonic Operators Become Full-Harmonic Oscillators after the Wall Slides Away. arXiv: 2108.00289.

[4] Fantoni, R. (2021) Monte Carlo Evaluation of the Continuum Limit of $\varphi_{3}^{12}$. Journal of Statistical Mechanics. Theory and Experiment, 2021, arXiv: 2011.09862. https://doi.org/10.1088/1742-5468/ac0f69

[5] Freedman, B., Smolensky, P. and Weingarten, D. (1982) Monte Carlo Evaluation of the Continuum Limit of $\varphi_{4}^{4}$ and $\varphi_{3}^{4}$. Physics Letters $B, 113,481-486$. https://doi.org/10.1016/0370-2693(82)90790-0

[6] Aizenman, M. (1981) Proof of the Triviality of $\varphi_{d}^{4}$ Field Theory and Some Mean-Field Features of Ising Models for $d>4$. Physical Review Letters, 47, 886. https://doi.org/10.1103/PhysRevLett.47.1

[7] Fröhlich, J. (1982) On the Triviality of $\lambda \varphi_{d}^{4}$ Theories and the Approach to the Critical Point in $d \geq 4$ Dimensions. Nuclear Physics B, 200, 281-296. https://doi.org/10.1016/0550-3213(82)90088-8

[8] Fantoni, R. and Klauder, J. (2021) Affine Quantization of $\varphi_{4}^{4}$ Succeeds While Canonical Quantization Fails. Physical Review D, 103, Article ID: 076013. https://doi.org/10.1103/PhysRevD.103.076013

[9] Klauder, J. (2021) Evidence for Expanding Quantum Field Theory. Journal of High Energy Physics, Gravitation and Cosmology, 7, 1157-1160. 
https://doi.org/10.4236/jhepgc.2021.73067

[10] Klauder, J. (2021) Using Coherent States to Make Physically Correct Classical-toQuantum Procedures That Help Resolve Nonrenomalizable Fields Including Einstein's Gravity. Journal of High Energy Physics, Gravitation and Cosmology, 7, 1019-1026. https://doi.org/10.4236/jhepgc.2021.73060

[11] Klauder, J. (2020) Using Affine Quantization to Analyze Nonrenotmalizable Scalar Fields and the Quantization of Einstein's Gravity. Journal of High Energy Physics, Gravitation and Cosmology, 6, 802-816. https://doi.org/10.4236/jhepgc.2020.64053

[12] Arnowitt, R., Deser, S. and Misner, C. (1962) The Dynamics of General Relativity. In: Witten, L., Ed., Gravitation: An Introduction to Current Research, Wiley \& Sons, New York, p. 227.

[13] Wikipedia. Ricci Scalar. https://en.wikipedia.org/wiki/Ricci_curvature

[14] Klauder, J. and Skagerstam, B.-S. (1985) Coherent States: Applications in Physics and Mathematical Physics. World Scientific, Singapore.

https://doi.org/10.1142/0096

[15] Klauder, J. (2006) Fundamentals of Quantum Gravity. arXiv: gr-qc/0612168v1. 